\title{
UCRL-ID-155334
}

AA WAE NOE IIVEAMOAE WATIONAL LAEXSATCOYY
Mark-1 Cryostat Status Report Q1 and Q2, FY03

T. P. Bernat

July 1, 2003 


\section{Mark-1 Cryostat Status Report Q1 and Q2, FY03}

Questions can be addressed to T. Bernat, bernat2@IInl.gov, 9254225915

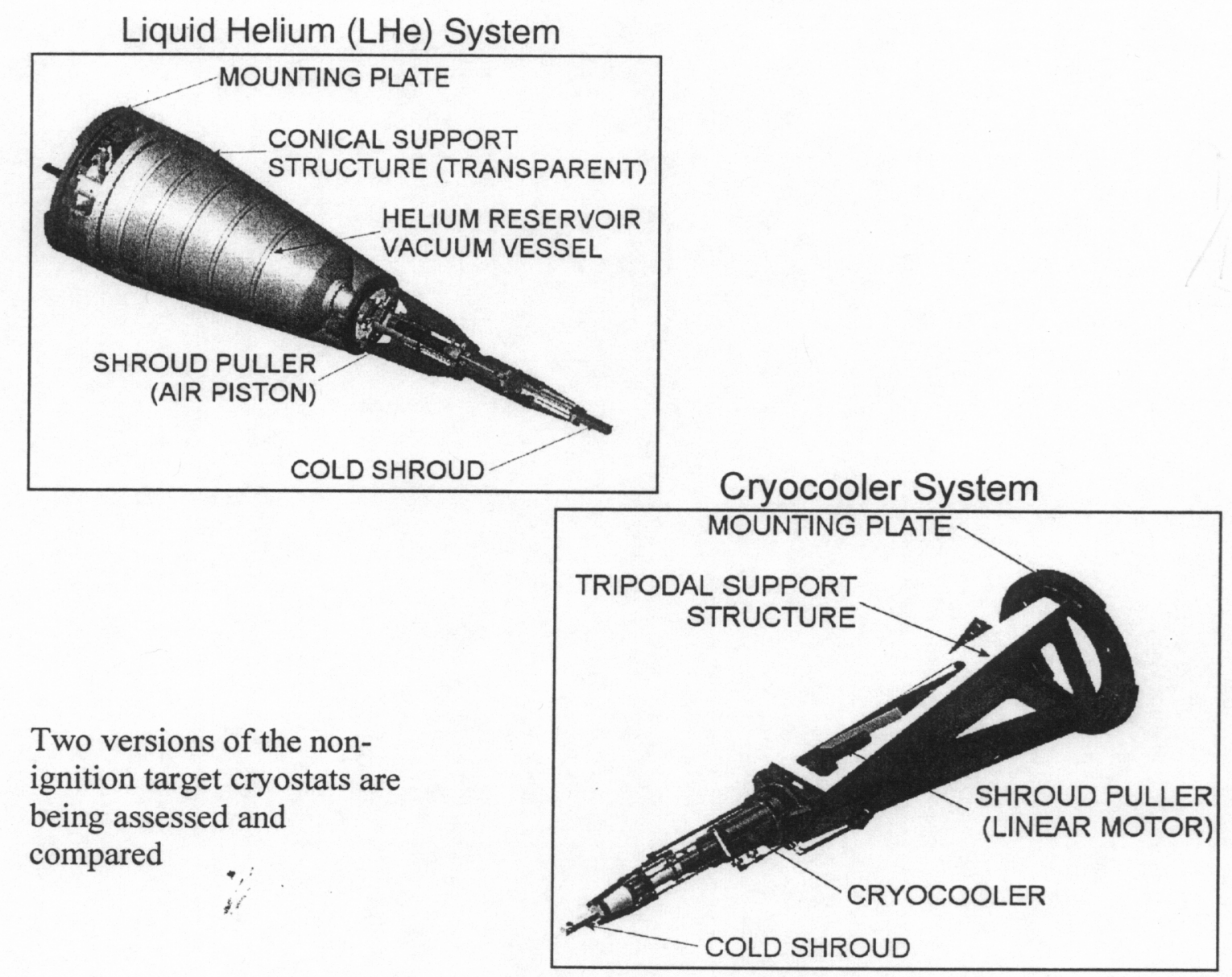

July 2003

Lawrence Livermore

National Laboratory

7000 East Avenue

Livermore, CA 94550

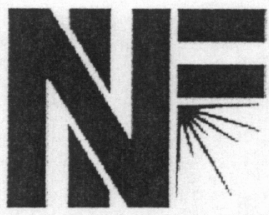


DISCLAIMER

This document was prepared as an account of work sponsored by an agency of the United States Government. Neither the United States Government nor the University of California nor any of their employees, makes any warranty, express or implied, or assumes any legal liability or responsibility for the accuracy, completeness, or usefulness of any information, apparatus, product, or process disclosed, or represents that its use would not infringe privately owned rights. Reference herein to any specific commercial products, process, or service by trade name, trademark, manufacturer, or otherwise, does not necessarily constitute or imply its endorsement, recommendation, or favoring by the United States Government or the University of California. The views and opinions of authors expressed herein do not necessarily state or reflect those of the United States Government or the University of California, and shall not be used for advertising or product endorsement purposes.

This report has been reproduced directly from the best available copy. Available to DOE and DOE contractors from the Office of Scientific and Technical Information P.O. Box 62, Oak Ridge, TN 37831

Prices available from (615) 576-8401, FTS 626-8401

Available to the public from the

National Technical Information Service

U.S. Department of Commerce

5285 Port Royal Rd.,

Springfield, VA 22161

This work was performed under the auspices of the U.S. Department of Energy, National Nuclear Security Administration by University of California Lawrence Livermore National Laboratory under Contract W-7405Eng-48. 


\section{Table of Contents}

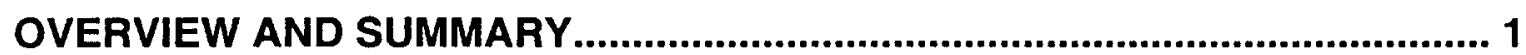

MARK-1 PROGRESS AND STATUS ........................................................... 1

Helium Reservoir Version ....................................................................................................................................................2

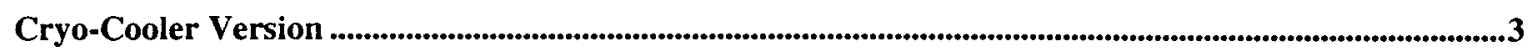

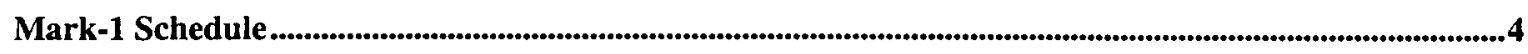

Risk Reduction and Concept Testing...............................................................................................................................5

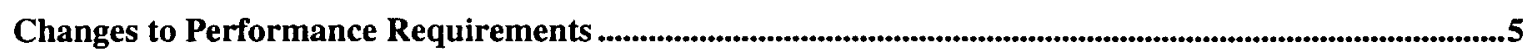




\section{Mark-1 Cryostat Status Report Q1 and Q2, FY03}

\section{Overview and Summary}

The Mark 1 now has a completion date goal of mid-FY05. The original concept for the Mark-1 cryogenics was based upon a liquid helium (LHe) reservoir, which was developed in collaboration with a group of cryogenic experts from Commissariat a L'Energie Atomique (CEA) in France. This concept meets all requirements and a conceptual design review was held in November 2002. However, meeting the completion date goal would be very difficult with this approach. Subsequent interaction with colleagues at the University of Rochester Laboratory for Laser Enegetics (URLLE) generated a modified concept with the LHe cryogenics replaced with a commercial mechanical cryo-cooler. This offers ease of operation, reduced cost, and a shorter schedule. However, some functional requirements cannot be met, and change requests are being written for review and acceptance. A high-level schedule for the cryo-cooler-based Mark-1 has been created, with a more detailed plan for the risk reduction and concept/component testing phase, which extends into the first half of FY04. A complete detailed schedule is in preparation.

\section{Mark-1 Progress and Status}

A requirements document for the Mark-1was prepared by NCTS staff with input from users at all inertial confinement fusion/high-energy-density science (ICF/HEDS) institutions. The document includes both functional requirements and engineering and ES\&H standards. The requirements were reviewed and approved by a committee chaired by Dan Kalantar (LLNL), comprising representatives from all ICF/HEDS institutions and user groups. The requirements were approved in October 2002. A document identifying all Mark-1 internal and external interfaces was also written and distributed.

In FY2002 and early FY2003, a more detailed design concept was developed for the Mark-1 based upon the General Atomics report "Conceptual Design Report of a NIF Mk-1 Cryogenic Target System" (GA-C23580, November 2000). This engineering concept used cold cryogen drawn from a liquid helium reservoir to cool targets and regulate their temperatures. This formed the basis for a Conceptual Design Review held in November 2002 at LLNL with video conference links to all the ICF/HEDS labs and to DOE/NNSA. The Conceptual Design Review committee was chaired by Bob Murray (LLNL) and was comprised of members of LLNL's engineering, ES\&H, and Quality Assurance staffs, plus representatives of each of the ICF/HEDS labs. Discussion during the CDR presentations generated a modification to the Mark-1 concept, using a commercial mechanical cryo-cooler to supply the cryogenics rather than the liquid helium reservoir. This concept was more fully developed in the second quarter of FY03.

Both Mark-1 cryostat concepts have their shapes designed to avoid diagnostic and optical interference in the NIF Target Chamber. They both fit into the NIF target positioner (TARPOS), 
replacing a carbon cone that is in use at present to hold non-cryogenic targets. They also both use the same type of "clam shell" target shroud that was reviewed previously.

\section{Helium Reservoir Version}

We began a collaboration with System des Basses Temperatures (SBT) in Grenoble, France, part of the French CEA, to take advantage of their expertise in cryogenic engineering. They are the primary designers and implementers of cryogenic target systems for the French Laser Megajoule project. Our primary interest is to have them do a more detailed design of the LHe reservoir section of the Mark-1 and to construct and test a prototype. Several NCTS staff visited SBT in November of 2002 to discuss and agree on the approach and sharing of responsibility for this project. It was agreed that NCTS staff would have responsibility for the "front-end" design, while SBT would do the "back end". NCTS staff would supply design constraints and design details of the back-end coupling to the NIF TARPOS.

Figure 1 shows the resulting Mark-1 design. Liquid helium flowing from the reservoir cools the cold head of the cryostat, to which the target is attached. Thermal analysis shows that the 36-liter reservoir should provide 1 Watt cooling to the target assembly at 5 Kelvins for 10 hours. After 10 hours, the reservoir must be refilled. Transient analysis shows that the cold tip continues to move at the rate of 1 micron per minute, even 80 minutes after the start of front-end cool down, which could interfere with the desired shot turn-around time. Further input from Bob Early at URLLE, based upon their planar target cryostat, allowed considerable simplification of the mechanism for attaching targets to the cold tip. The mechanism is shown in Figure 2. Targets are placed on the cold tip in warm state and secured with the aluminum clamping nut. Differential thermal contraction between aluminum and copper serves to tighten the contact between the target base and the cold tip, thus ensuring good thermal contact. This concept has been tested and is in use at URLLE.

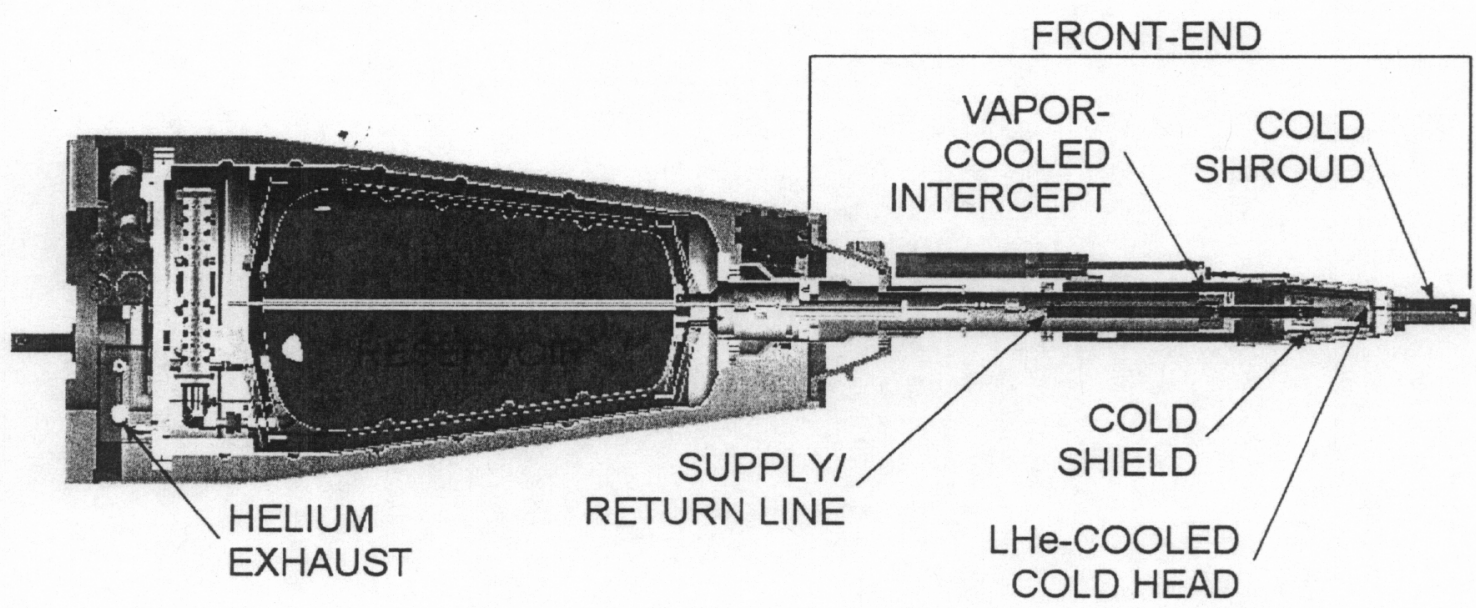

Figure 1. The liquid helium reservoir version of the Mark-1. 


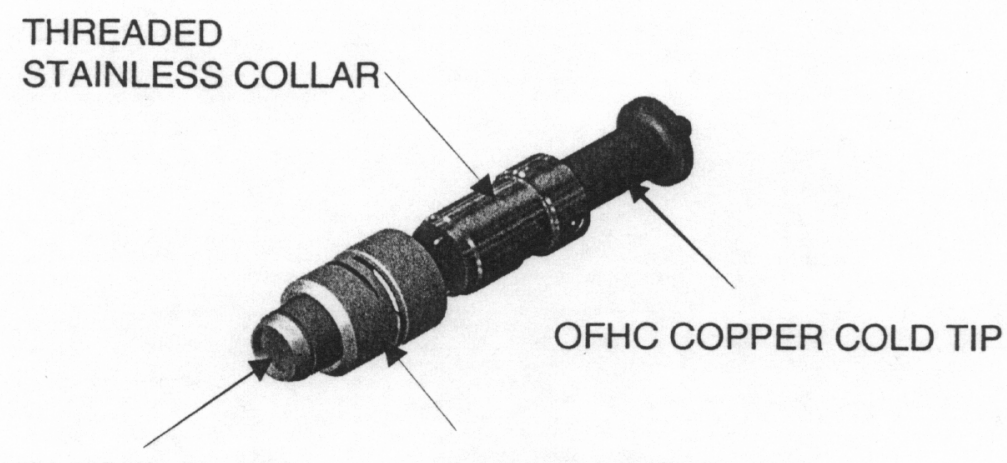

TARGET BASE

AL CLAMPING NUT

Figure 2. Simplified Mark-1 target mounting arrangement, based upon designs at URLLE.

\section{Cryo-Cooler.Version}

In December 2002, NCTS staff visited the University of Rochester Laboratory for Laser Energetics to review their approach to fielding a simple cryostat for performing planar shock and equation of state experiments on the Omega facility. While the system constraints between Omega and the NIF are considerably different, and the requirements for the Mark-1 are not the same as for LLE's planar cryostat, it was readily apparent that using a commercially available mechanical cryo-cooler could provide simplification of use and lower operational costs, as well as lower acquisition costs with a shorter acquisition schedule. We therefor, developed and began analyzing a design concept for the Mark-1 based upon the cryo-cooler. The design concept is shown in Figure 3.

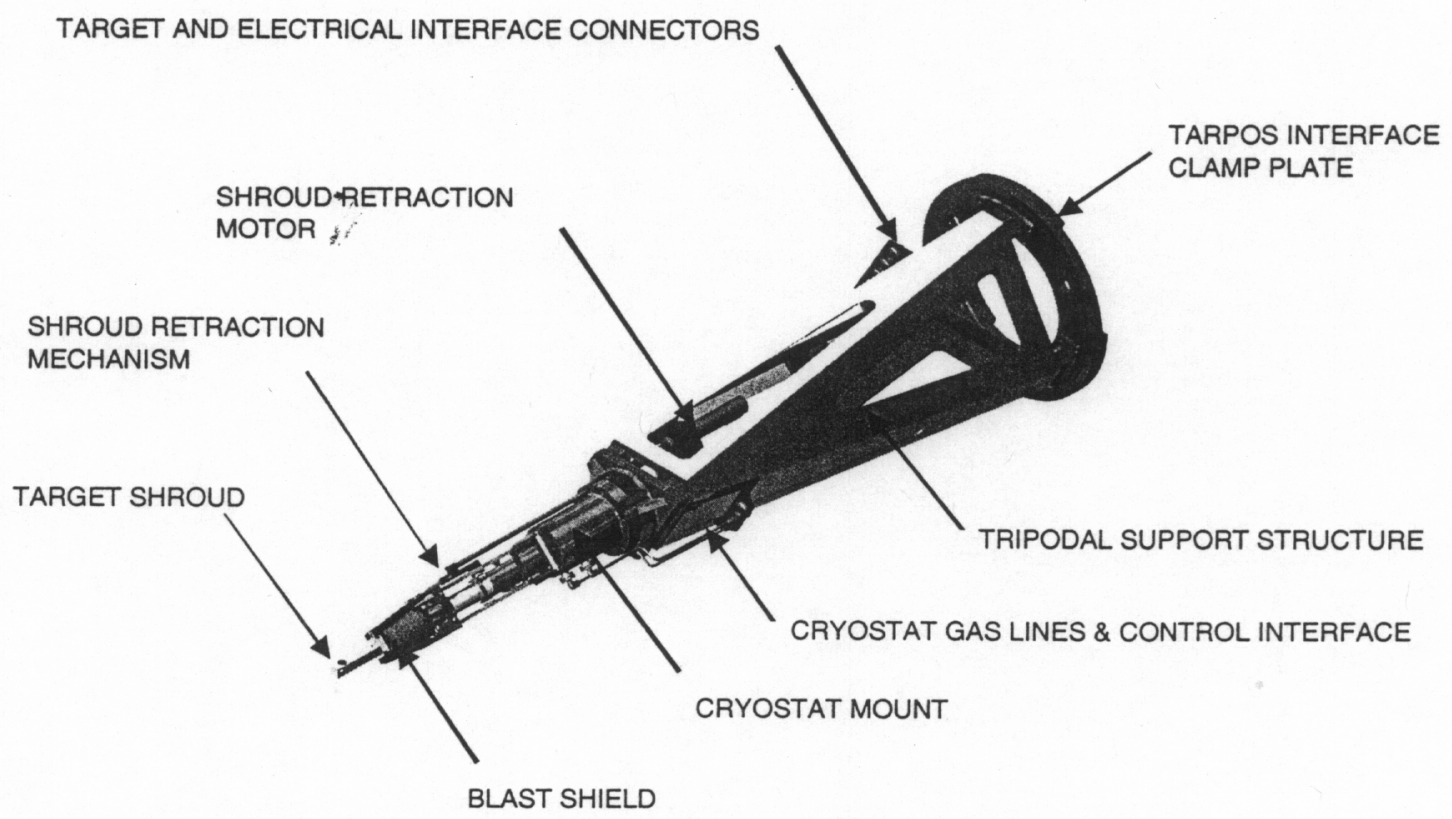

Figure 3. Design concept for a cryo-cooler based Mark-1 cryostat. 
Compared to the reservoir-based concept in Figure 6, the cryo-cooler-based design is lighter and more open in the back, greatly facilitating the placement of cables, gas lines and the shroud actuator, as well as attachment to the TARPOS mount flange. The use of the cryo-cooler makes helium refilling and recovery unnecessary, which will greatly reduce operational costs. The cryo-cooler can maintain a target cold indefinitely, so interruptions to shot schedules from any NIF system problems can be easily accommodated.

The use of a cryo-cooler, however, yields a Mark-1 that does not exactly meet three of the operational requirements, although the differences are not large. First, the lowest temperature at the target mounting cold base is $7.5 \mathrm{~K}$ rather than $5 \mathrm{~K}$, with a heat load from the target assembly of 1 Watt. The base can reach $5 \mathrm{~K}$, but only with 0.3 Watts from the target assembly. Second, the target mount base will oscillate in the direction of the TARPOS axis with a $25-\mu \mathrm{m}$ amplitude at $2.5 \mathrm{~Hz}$. If the target assembly is rigidly attached to the target base, it will experience this same motion. Experience at URLLE has shown that this motion stops after turning off the cryo-cooler in a short enough time that the target assembly has not started to warm appreciably. We will examine this with a bench-level test system in the second half of FY03. And third, the cooldown time for the cryo-cooler based system will be close to 60 minutes. This may affect the desired ability for 2 shots in one 8 -hour period.

\section{Mark-1 Schedule}

A high-level schedule based upon estimates of durations for the various engineering phases of the Mark-1 is shown in Figure 4. Component development and testing started at a low level in FY02, during which we tested a pneumatic shroud actuator, and designed equipment to test the temperature stability and cooling power of a commercial liquid helium flow cryostat line. We also worked with SBT, Grenoble, colleagues to define a prototype Mark-1 based upon a liquid helium flow cryostat, as described above. In FY03 we began work on the cryo-cooler version, and the prototyping and testing associated with this is discussed in the section below. To complete and activate the Mark-1 on this ambitious schedule demands that phases are done somewhat in parallel. This is possible because we anticipate that many of the components produced for the prototyping and testing phase will ultimately comprise parts of the final system.

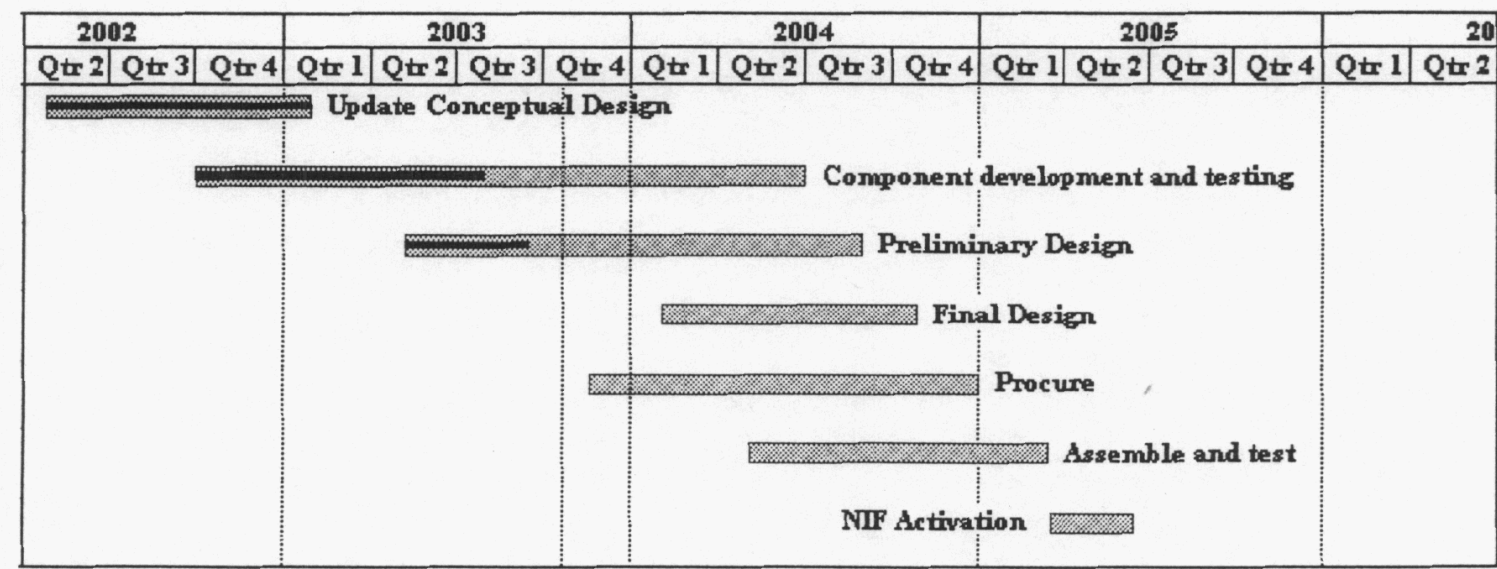

Figure 4. High-level schedule for the Mark-1 with a commercial cryo-cooler. 


\section{Risk Reduction and Concept Testing}

Engineering solutions to several key requirements must be verified through a combination of computer modeling and component prototyping and testing, prior to completing the preliminary design of the Mark-1. First, we must assure that the cryo- cooler operation spans the entire temperature range required, with the required stability. We will be using the cryo-cooler in a non-normal mode at the higher temperatures, which may require a shunt valve on the highpressure helium circuit. Such a shunt valve is used at URLLE. Second, we want to verify cooldown times and the warm-up curve upon turning off the cryo-cooler. We will also check to see how long it takes to stabilize the tip position, as well as the repeatability of this position, after turning off the cryo-cooler. Third, we must construct and test a first version of the shroud and its retraction mechanism, and make sure the shroud retraction does not disturb the target position. And fourth, we must select high-pressure helium lines for the cryo-cooler with good flexibility, and test these for outgassing, since they will be inside the NIF chamber. The schedule for this work phase is shown in Fig. 5. The initial set of thermal and mechanical tests for the shroud and actuators are scheduled to be complete by the end of Q1FY04, but extend to the end of Q2 to allow for modifications and retesting.

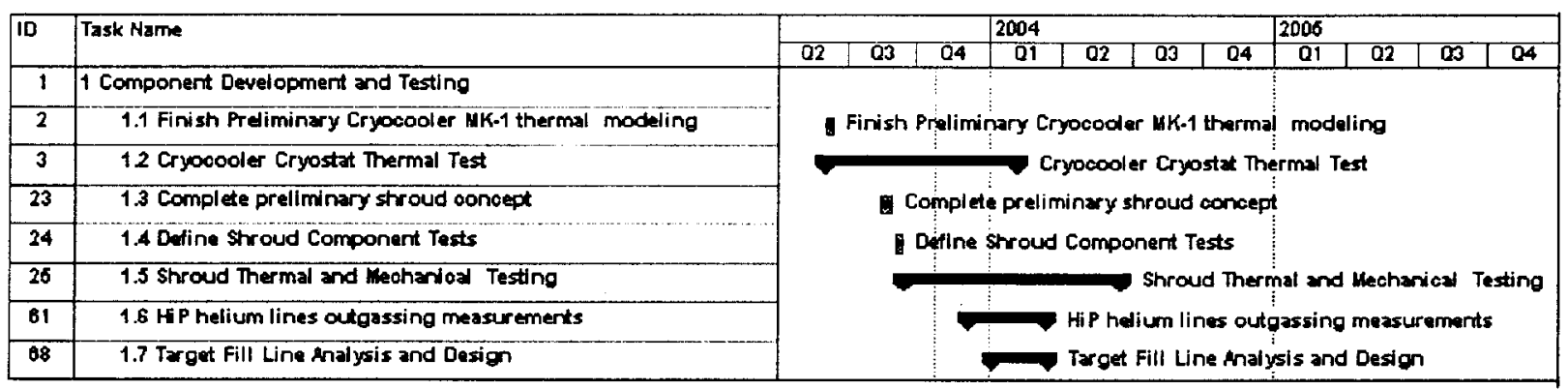

Figure 5. Schedule for the component development and testing phase of the Mark-1 cryostat.

\section{Changes to Performance Requirements}

Three changes to the performance requirements for the Mark 1 cryostat are being evaluated. Two of these changes are to accommodate the use of the commercial cryo-cooler: raising the lowest temperature for the target assembly base attachment point to the Mark-1 cryostat from $5 \mathrm{~K}$ to 7.5 $\mathrm{K}$, at 1 Watt cooling power, and allowing the $25-\mu \mathrm{m}$ oscillatory motion due to operation of the cryo-cooler. The third change adds a requirement for accommodating neutron yields up to $10^{16}$ neutrons while maintaining occupational activation dose limits for the NIF and for target area operations. These changes will be discussed with Mark 1 users from the ICF/HEDS laboratories. 